\title{
Treatment of Cluster Headache in a Different Light: A Case Study
}

\author{
Arnold Wilkins and Nicholas Cooper \\ Department of Psychology, University of Essex, Colchester, UK
}

W e report on a patient with cluster headache who wore spectacles tinted to provide maximum comfort when reading printed text. The spectacles absorbed short-wavelength light and were only worn during the prodrome period. The patient has been free of cluster headaches for the 6 years since the spectacles became available, and the prodromes have decreased in severity and frequency. The hypothalamus is highly active during cluster headaches and receives visual input from the intrinsically photosensitive retinal ganglion cells (ipRGCs). The ipRGCs are most sensitive to short-wavelength light.

\section{Keywords}

Cluster headache, precision tints, green light, ipRGCs, prodrome

Disclosures: Arnold Wilkins invented the Intuitive Colorimeter ${ }^{\mathrm{TM}}$. He has donated all emoluments to the University of Essex for use in student bursaries. Nicholas cooper has no financial or non-financial relationships or activities to declare in relation to this article.

Review process: Double-blind peer review.

Compliance with ethics: Informed consent was received from the patient involved in this case study, and no identifying information or images have been included. Data availability: The datasets generated during and/ or analysed during the current study are available from the corresponding author on reasonable request. Authorship: The named authors meet the International Committee of Medical Journal Editors (ICMJE) criteria for authorship of this manuscript, take responsibility for the integrity of the work as a whole, and have given final approval for the version to be published.

Access: This article is freely accessible at touchNEUROLOGY.com (c) Touch Medical Media 2021

Received: 9 September 2021

Accepted: 19 October 2021

Published online: 13 December 2021

Citation: touchREVIEWS in Neurology 2021;17(2): 110-11

Corresponding author: Arnold Wilkins, Department of Psychology, University of Essex, Colchester, CO4 3SQ, UK. E: Arnold@essex.ac.uk

Support: No funding was received in the publication of this article.
A university lecturer first experienced headaches during the summer of 1989, when he was aged 26 years. The headache periods initially lasted about 1 day and because the pain was in the trigeminal area both an optometrist and a dentist were consulted. The headaches gradually increased in length, severity and frequency over the ensuing 10 years, typically lasting 4-6 weeks every other year, and always in late spring or early summer. Each headache typically lasted 45 90 minutes and occurred 3-5 times per day. The episodes, now recognized as cluster headache, were preceded by 2-4 weeks of prodromes, known to the patient as 'shadows'. The prodromes manifested as: 1) a unilateral feeling of pressure over the right trigeminal area (same side as the cluster headache pain) that could progress through ache to pain of moderate to severe intensity; 2) occasional feeling of blocked nasal passage (right side); 3) occasional mild neck pain. The symptoms could last from a few hours to an entire day, and built up in frequency and intensity over the course of about a month before the onset of the cluster headache period. Atypically, the prodrome also occurred in December of each year, but did not then develop into episodes of cluster headache. Occasionally, the patient could stop a prodrome from developing into a cluster using oral sumatriptan, but only rarely. The cluster headaches continued unaltered for 13 years until the patient was seen in 2002 at the National Hospital for Neurology and Neurosurgery, London, UK. A computed tomography scan and neurological examination were unremarkable. Pain management with subcutaneous sumatriptan was initiated and, in 2006, this was supplemented with oxygen. The patient has no other health issues. Both the patient's parents had migraine.

In 2014, the patient was given a pair of light-weight spectacles with precision tints as part of a research project at his university. ${ }^{1}$ The chromaticity of the lenses was selected using the Intuitive Colorimeter ${ }^{\top M}$ (Cerium Visual Technologies Ltd., Tenterden, UK). ${ }^{1.2}$ He chose a chromaticity (yellowgreen) that best improved the clarity of and comfort when reading a page of text, and spectacle lenses were then made that provided this chromaticity under white (F2) fluorescent light. The spectral transmission of the lenses is shown in Figure 1. The lenses transmitted $56 \%$ of the light and their Uniform Chromaticity Scale chromaticity was $u^{\prime}=0.189, v^{\prime}=0.529$.

The patient discovered that his prodrome did not progress to a cluster headache when he was using the tinted spectacle lenses. When he put the spectacles on, the sensations of pressure and mild pain gradually dissipated rather than rising to the excruciating piercing pain of cluster headache. Between 2014 and 2021, he was able to avert innumerable prodromes by wearing the tinted lenses; he does not wear them at other times. Since the tinted spectacles were provided, he has not experienced a cluster headache and has not used sumatriptan and oxygen. Over these 6 years, the symptoms have become less pronounced and quicker to stop. By 2021, the prodromes had decreased to about 2 per year. Consent for publication was provided by the patient. The research project was approved by the University of Essex Ethics Committee, reference AW1203.

\section{Discussion}

Cluster headache is a disease of long duration and, when episodic, it tends to worsen, particularly in male patients with a disease duration of over 20 years. ${ }^{3}$ In this case, however, the abrupt cessation of cluster attacks when the spectacles were first made available and the failure of repeated prodromes to progress to cluster headaches when the tinted spectacles were worn both suggest 
Figure 1: Spectral transmission of the tinted glasses

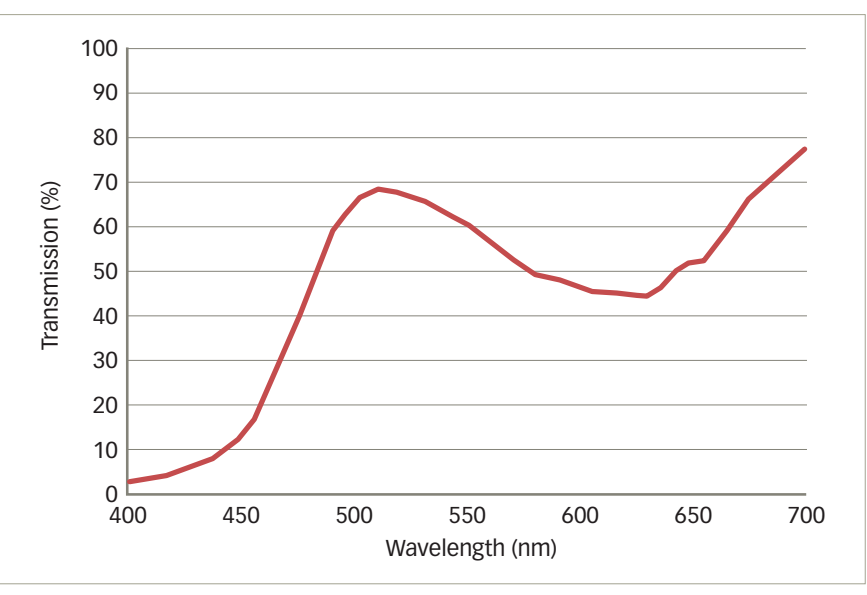

that the tint was preventing the progression of the cluster headache. The precision tint alleviated discomfort in and around the eye - the same location as the pain at the start of the cluster headache. The decrease in the frequency of prodromes suggests that the beneficial effect of the tint was not simply the prevention of the prodrome developing into an attack. Other longer-term benefits also accrued, possibly as a result of the abolition of headaches; spontaneous improvement may also have been a factor.

In general, the light people choose as being comfortable for reading has a chromaticity close to the Planckian locus (i.e. similar to the various colours of daylight). ${ }^{4,5}$ In migraine with aura, however, the comfortable chromaticity lies distant from the Planckian locus and the colour is strongly saturated, ${ }^{4,5}$ as in this case. The tinted spectacles were yellow-green. Green light is better tolerated than light of other colours when the light is sufficiently bright to cause symptoms. ${ }^{6}$ The spectral transmission of the tint (Figure 1) absorbed short-wavelength light, where the intrinsically photosensitive retinal ganglion cells (ipRGCs) are most sensitive, with possible consequence for the pupillary response ${ }^{7}$ and light sensitivity. ${ }^{8}$ Neuroimaging studies have found that the hypothalamus is hyperactive during cluster headache attacks. ${ }^{9,10}$ The hypothalamus is known to have a visual input from the ipRGCS ${ }^{11,12}$ and may be involved in the circadian and seasonal nature of cluster headaches ${ }^{13,14}$ evident in this case.

The effects of the tinted spectacles may have occurred because their spectral transmission was individually selected for maximum comfort and/or because they attenuated short-wavelength light. Vingen et al. ${ }^{15}$ found a self-reported frequency of photophobia in $91 \%$ of 50 patients with cluster headache, and about $20 \%$ of patients report bright or flashing light as triggers. ${ }^{16}$ The Intuitive Colorimeter allows an ophthalmic tint to be designed individually to reduce photophobia. 1,2,17,18

Photophobia may be an important component of the prodrome in cluster headache, and when treated, the reduction in photophobia can sometimes evidently prevent the progression to cluster headache. As a single case report, this study has obvious limitations, and it remains to be seen whether other patients with cluster headache can benefit from precision tints. $\square$
1. Wilkins AJ, Sihra N. A colorizer for use in determining an optimal ophthalmic tint. Color Res Appl. 2001;26:246-53.

2. Wilkins AJ. Visual stress: origins and treatment. CNS. 2021;6: $1-13$

3. Manzoni GC, Micieli G, Granella F, et al. Cluster headache - course over ten years in 189 patients. Cephalalgia. 1991:11:169-74.

4. Aldrich A, Hibbard P, Wilkins A. Vision and hyper-responsiveness in migraine. Vision (Basel). 2019:3:62

5. Vieira A, van der Linde I, Bright P, Wilkins A. Preference for lighting chromaticity in migraine with aura. Headache. for lighting chrom

6. Noseda R, Bernstein CA, Nir RR, et al. Migraine photophobia originating in cone-driven retinal pathways. Brain. 2016;139:1971-86

7. MCDougal DH, Gamlin PD. The influence of intrinsicallyphotosensitive retinal ganglion cells on the spectral sensitivity and response dynamics of the human pupillary light reflex. Vision Res. 2010;50:72-87.

8. Noseda R, Copenhagen D, Burstein R. Current understanding of photophobia, visual networks and headaches. Cephalalgia. 2019;39:1623-34

9. May A, Bahra A, Büchel C, et al. Hypothalamic activation in cluster headache attacks. Lancet. 1998;352:275-8.

10. Qiu E, Wang Y, Ma L, et al. Abnormal brain functional connectivity of the hypothalamus in cluster headaches. PLOS Connectivity of the hy
One. 2013;8:1-8.

11. Baver SB, Pickard GE, Sollars PJ, Pickard GE. Two types of melanopsin retinal ganglion cell differentially innervate the hypothalamic suprachiasmatic nucleus and the olivary pretectal nucleus. Eur J Neurosci. 2008;27:1763-70.

12. Li JY, Schmidt TM. Divergent projection patterns of M1 IPRGC subtypes. J Comp Neurol. 2018;526:2010-18.

13. Nishino $\mathrm{H}$, Kiyomi $\mathrm{K}$, Brooks $\mathrm{CM}$. The role of suprachiasmatic nucleus of the hypothalamus in the production of circadian rhythm. Brain Res. 1976;112:45-59.

14. Leone H, Franzini A, Broggi G, Bussone G. Hypothalamic stimulation for intractable cluster headache: long-term experience. Neurology. 2006;67:150-2

15. Vingen JV Pareja JA, Stovner II. Quantitative evaluation of photophobia and phonophobia in cluster headache. Cephalalgia. 1998;18:250-6.

16. Rozen T, Fishman RS. Cluster headache in the United States of America: demographics, clinical characteristics, triggers, suicidality and personal burden. Headache. 2012:5:99-113.

17. Wilkins AJ, Haigh SM, Mahroo O, Plant GT. Photophobia in migraine: a symptom cluster. Cephalalgia. 2021;41:1240-8.

18. Aldrich $A$, Lovell-Patel $R$, Allen $P$, Wilkins AJ. The repeatability of colorimetry is precise(ly) as expected. Neuro-ophthalmology \& Visual Neuroscience. 2018;3:1-6. 